\title{
LAX EQUATIONS, SINGULARITIES AND RIEMANN-HILBERT PROBLEMS
}

\author{
ANTÓNIO F. DOS SANTOS AND PEDRO F. DOS SANTOS
}

\begin{abstract}
The existence of singularities of the solution for a class of Lax equations is investigated using a development of the factorization method first proposed by Semenov-Tian-Shansky and Reymann [11, 9 9. It is shown that the existence of a singularity at a point $t=t_{i}$ is directly related to the property that the kernel of a certain Toeplitz operator (whose symbol depends on $t$ ) be non-trivial. The investigation of this question involves the factorization on a Riemann surface of a scalar function closely related to the above-mentioned operator. An example is presented and the set of singularities is shown to coincide with the set obtained by classical methods. This comparison involves relating the two Riemann surfaces associated to the system by these methods.
\end{abstract}

\section{INTRODUCTION}

In this paper we investigate the existence of singularities of the solutions of Lax equations for a class of equations that applies to most finite-dimensional dynamical systems such as e.g. classical tops (see e.g. [3], 9], [10]). To that end we consider the time variable $t$ to be a complex variable and determine the singularities of the solution in the complex plane. This is tied to the question of global existence of solutions for real $t$ as the non-existence of singularities for real $t$ implies global existence of the solution. Also, it is likely that full knowledge of the location of complex singularities may eventually give more insight into the dynamics of the system.

Our approach is a development of the factorization method first proposed by Semenov-Tian-Shansky and Reymann [11, [9] which in turn may be seen as a generalization of the AKS (Adler-Kostant-Symes) theorem that applies to finite dimensional algebras [1].

To the best of our knowledge the first application of this method in the setting of an infinite-dimensional algebra appeared in [4], which focused on a restricted class of Lax equations. The absence in the literature of more fully computed examples of application of this method is probably due to the fact that it involves the factorization of a continuous function on a contour in a Riemann surface (for a general treatment of this problem see [5]).

Considering $t$ as a complex variable and extending the class of Lax equations requires a new analysis of the results of [4], where we were able to avoid making some delicate assumptions like the differentiability 
of the factors in the Wiener-Hopft factorization (see definition below) of the matrix function $\exp \left(t L_{0}\right)$, where $L_{0}$ is the value of the Lax matrix $L_{t}$ at $t=0$. This is done in Section 2 and continued in Section 3 for the question of location of the singularities of the solution. Our approach makes the treatment fully rigorous and, in our view, is crucial for the treatment in the context of a complex $t$.

The main result is Theorem 3.1, which we state next. For this we note that the space $\left[C_{\mu}\left(S^{1}\right)\right]^{n}$ of Hölder continuous $n \times n$ matrix-valued functions has a direct sum decomposition

$$
\left[C_{\mu}\left(S^{1}\right)\right]^{n}=\left[C_{\mu}^{+}\right]^{n} \oplus\left[C_{\mu}^{-}\right]_{0}^{n},
$$

where $\left[C_{\mu}^{+}\right]^{n}$ is the subspace 11 of functions having an analytic extension to the unit disc $\mathbb{D}$ and $\left[C_{\mu}^{-}\right]_{0}^{n}$ is the subspace of functions admitting analytic extensions to $\mathbb{C} \backslash \overline{\mathbb{D}}$ that vanish at infinity. In what follows $\left[C_{\mu}^{-}\right]^{n}=\left[C_{\mu}^{-}\right]_{0}^{n} \oplus \mathbb{C}^{n}$.

Consider the Toeplitz operator

$$
T_{G}=P^{+} G I_{+}:\left[C_{\mu}^{+}\right]^{n} \rightarrow\left[C_{\mu}^{+}\right]^{n}
$$

where $G=\exp \left(t L_{0}\right), I_{+}$is the identity operator on $\left[C_{\mu}^{+}\right]^{n}$ and $P^{+}$ is the projection of $\left[C_{\mu}\right]^{n}$ onto $\left[C_{\mu}^{+}\right]^{n}$ associated to the decomposition (1.2). Theorem 3.1 states that the Lax equation,

$$
\frac{d L_{t}}{d t}=\left[L_{t}^{+}+A_{0}, L_{t}\right]
$$

has a solution in a neighbourhood of the point $t_{i}$ iff $T_{G}$ is injective at the point $t_{i}$. In the above equation $A_{0}=P_{0} L_{t}$ where $P_{0}:\left[C_{\mu}\left(S^{1}\right)\right]^{n \times n} \rightarrow$ $\mathbb{C}^{n \times n}$ is a bounded linear operator.

In the calculation of the singularities we need the notion of a WienerHopf factorization. Let $G: S^{1} \rightarrow\left[C_{\mu}\right]^{n \times n}$. We say that $G$ possesses a Wiener-Hopf factorization (also called Riemann-Hilbert factorization and Birkhoff factorization [7], [8]) if $G$ can be represented in the form

$$
G=G_{-} D G_{+},
$$

where $G_{ \pm}$and their inverses belong to $\left[C_{\mu}^{ \pm}\right]^{n \times n}$ and $D=\operatorname{diag}\left(r^{k_{1}}, \ldots, r^{k_{n}}\right)$ with $k_{1} \geq k_{2} \geq \cdots k_{n}$ and $r$ is a rational function with a zero in $\mathbb{D}$ and a pole in $\mathbb{C} \backslash \overline{\mathbb{D}}$. The factorization is said to be canonical if $D=I_{n}$, where $I_{n}$ denotes the identity matrix. The above definition applies to functions belonging to other spaces (see e.g. [6]). In $\left[C_{\mu}\right]^{n \times n} G$ possesses a Wiener-Hopf factorization (1.3) iff $G$ is invertible on $S^{1}$. We recall from [6] that the operator $T_{G}$ is invertible iff the factorization (1.4) is canonical. This is the basic result from operatior theory that will be used to locate the singularities of the solution of the Lax equations. A direct consequence of Theorem 3.1 is Proposition 3.2 which

\footnotetext{
${ }^{1}$ We have omitted $S^{1}$ to simplify notation
} 
states that the solution of equation (1.3) has a singularity at $t=t_{i}$ iff the Riemann-Hilbert problem

$$
G \Phi^{+}=\Phi^{-} \quad\left(\Phi^{+} \in\left[C_{\mu}^{+}\right]^{n}, \Phi^{-} \in\left[C_{\mu}^{-}\right]_{0}^{n}\right)
$$

has a nontrivial solution at $t=t_{i}$. This is equivalent to saying that the Wiener-Hopf factorization of $G$ is noncanonical for $t=t_{i}$.

The paper ends with an example of a dynamical system that belongs to the standard Lax class considered in [4]. For this example it is possible to obtain the solution to the Lax equation by classical methods (integration of the system of ordinary differential equations) and thus obtaining its set of singularities. This enables us to compare it with the set of singularities derived by our method. A rather interesting point is that the classical approach and the Lax equation one lead to different Riemann surfaces. The two surfaces are closely related, as shown in Proposition 4.2, but the fact that they are different led us to derive several intermediate results in order to show that the sets of singularities obtained by the two approaches coincide.

The study of the example given in Section 4 takes a large part of the paper but we believe that it not only illustrates the theory that we present here but also sheds some light into the relation between the classical methods and those based on the Lax equation - a point that may be obscure in the study of other finite-dimensional integrable systems, for example, in the study of some classical tops.

\section{LAX EQUATION AND Riemann-Hilbert PROBlems}

In this section we generalize the results of [4, §2] replacing a neighborhood of the origin in the real variable $t$ by a neighborhood of the origin in the now complex variable $t$ (for what follows it is necessary to consider $t$ as complex variable) and extending the class of equations considered. In [4] we studied a class of Lax equations of the form

$$
\frac{d L_{t}}{d t}=\left[L_{t}^{+}, L_{t}\right]
$$

where the dynamical variables $L_{t}^{+}, L_{t}$ depend on a parameter $\lambda$ varying on the unit circle $S^{1}, L_{t}$ is a matrix-valued Laurent polynomial in $\lambda$ and $L_{t}^{+}$is the part of $L_{t}$ analytic in the unit disc $\mathbb{D}$. In [4] we called the above class the standard Lax class (it includes e.g. a special case of the Lagrange top). In this paper we study a class of Lax equations more general than the above one. It includes most finite-dimensional integrable systems. We write the equations of this class in the form

$$
\frac{d L_{t}}{d t}=\left[L_{t}^{+}+A_{0}, L_{t}\right]
$$


where $L_{t}^{+}$is defined as above and $A_{0}=P_{0} L_{t}$, with $P_{0}$ being a bounded linear operator from the space $\left[C^{1}(\Omega)\right]^{n \times n}$ of matrix-valued Hölder functions on $S^{1}$ to the space $\mathbb{C}^{n \times n}$ of constant matrix functions on $S^{1}$ (depending on $t$ as a parameter).

To state the first result in a rigorous way we need the definition that follows

Definition 2.1. Let $\left[C^{1}(\Omega)\right]^{n \times n}$ be the space of continuously differentiable matrix functions with respect to $t$ in a region $\Omega \subset \mathbb{C}$ and define $L_{t}(\lambda) \in\left[C^{1}(\Omega)\right]^{n \times n}$ to be a Laurent polynomial of the form

$$
L_{t}(\lambda)=\sum_{k=-m}^{1} L_{t}^{(k)} \lambda^{k} \quad\left(m \in \mathbb{N}, \lambda \in S^{1}\right),
$$

where $L_{t}^{(k)} \in \mathbb{C}^{n \times n}$. This gives for $L_{t}^{+}(\lambda)$ the expression

$$
L_{t}^{+}(\lambda)=\sum_{k=0}^{1} L_{t}^{(k)} \lambda^{k}
$$

Remark 2.2. In the case of the standard Lax class $\left(A_{0}=0\right.$ in (2.2) equation (2.2) together with formulas (2.3) and (2.4) imply that $L_{t}^{(1)}$ is a constant of the dynamics.

We can now state our first result which is a generalization of [4, Theorem 2.3] extending the applicability of known formulas (see e.g. [9]) for $L_{t}$.

Theorem 2.3. Let $L_{t}$ be an $n \times n$ matrix-valued function satisfying the Lax equation (2.2) in a simply-connected region $\Omega$ containing the origin (in the variable $t$ ). Then $L_{t}$ is given in the region $\Omega$ by the formulas

$$
L_{t}=\widetilde{G}_{+} L_{0} \widetilde{G}_{+}^{-1}=\widetilde{G}_{-}^{-1} L_{0} \widetilde{G}_{-}
$$

where $L_{0}=\left.L_{t}\right|_{t=0}$ and $\widetilde{G}_{+}, \widetilde{G}_{-}$satisfy in $\Omega$ the linear differential equations

$$
\frac{d \widetilde{G}_{+}}{d t}=\left(L_{t}^{+}+A_{0}\right) \widetilde{G}_{+} \quad \frac{d \widetilde{G}_{-}}{d t}=\widetilde{G}_{-}\left(L_{t}^{-}-A_{0}\right)
$$

subject to the initial conditions $\left.\widetilde{G}_{+}\right|_{t=0}=\left.\widetilde{G}_{-}\right|_{t=0}=I_{n}$, where $I_{n}$ is the identity $n \times n$ matrix.

Proof. The proof goes along the same lines as the proof of [4, Theorem $2.3]$ with $L_{t}^{ \pm}$replaced by $L_{t}^{ \pm} \pm A_{0}$. We note only that the condition on the connectivity of $\Omega$ is needed to ensure that equations (2.6) have well defined solutions throughout $\Omega$.

Proposition 2.4. If the singularities of $L_{t}$ with respect to $t$ are isolated then there exists a simply connected region $\Omega=\mathbb{C} \backslash B_{s}$ where $B_{s}$ is the union of two cuts joining the singularities. 
Proof. If the singularities are isolated we can denote them by $t_{n}$ with $n \in \mathbb{Z}$. Furthermore we can enumerate them in lexicographic order $\left(t \leq t^{\prime}\right.$ iff $\operatorname{Re} t<\operatorname{Re} t^{\prime}$ or $\operatorname{Re} t=\operatorname{Re} t^{\prime}$ and $\left.\operatorname{Im} t \leq \operatorname{Im} t^{\prime}\right)$. Consider two consecutive points of this sequence, say $t_{k}, t_{k+1}$. Define one cut $\left(B_{s^{+}}\right)$ as the union of line segments $\left[t_{r-1}, t_{r}\right]$ for $r<k$. Similarly, define the second cut $\left(B_{s^{-}}\right)$as the union of segments $\left[t_{r}, t_{r+1}\right]$ for $r \geq k$. Then $\mathbb{C} \backslash\left(B_{s^{+}} \cup B_{s^{-}}\right)$is simply connected.

Theorem 2.5. Let $G=\widetilde{G}_{-} \widetilde{G}_{+}$where $\widetilde{G}_{-}, \widetilde{G}_{+}$satisfy equations (2.6) in a simply connected region $\Omega$ containing the origin (in the variable $t$ ) and the condition $\left.G\right|_{t=0}=I_{n}$. Then

$$
G=\exp \left(t L_{0}\right)
$$

Proof.

$$
\begin{aligned}
\frac{d G}{d t} & =\frac{d \widetilde{G}_{-}}{d t} \widetilde{G}_{+}+\widetilde{G}_{-} \frac{d \widetilde{G}_{+}}{d t} \\
& =\widetilde{G}_{-}\left[\widetilde{G}_{-}^{-1} \frac{d \widetilde{G}_{-}}{d t}+\frac{d \widetilde{G}_{+}}{d t} \widetilde{G}_{+}^{-1}\right] \widetilde{G}_{+}
\end{aligned}
$$

From equations (2.6)

$$
\widetilde{G}_{-}^{-1} \frac{d \widetilde{G}_{-}}{d t}+\frac{d \widetilde{G}_{+}}{d t} \widetilde{G}_{+}^{-1}=L_{t}^{-}-A_{0}+L_{t}^{+}+A_{0}=L_{t}
$$

Then

$$
\frac{d G}{d t}=\widetilde{G}_{-} L_{t} \widetilde{G}_{+}=L_{0} G,
$$

where we have introduced the expression for $L_{0}$ resulting from the second of formulas (2.5),$L_{0}=\widetilde{G}_{-} L_{t} \widetilde{G}_{-}^{-1}$. Formula (2.7) now follows from the above equation.

\section{Theorem 2.6.}

(i) The factorization of $G=\exp \left(t L_{0}\right), G=\widetilde{G}_{-} \widetilde{G}_{+}$is a canonical Wiener-Hopf factorization in the region $\Omega$ of Theorem 2.5.

(ii) Let $G_{-} G_{+}$be another Wiener-Hopf factorization. Then

$$
\widetilde{G}_{+}=G_{+} F_{t}, \quad \widetilde{G}_{-}=G_{-} F_{t}^{-1} .
$$

Proof. (i) Let $\widetilde{G}_{-} \widetilde{G}_{+}$be the factorization of $G$ obtained in Theorem 2.5, i.e.

$$
G=\exp \left(t L_{0}\right)=\widetilde{G}_{-} \widetilde{G}_{+} .
$$

This is a canonical Wiener-Hopf factorization of $G$ in view of the properties of $\widetilde{G}_{-}, \widetilde{G}_{+}$resulting from equations (2.6).

(ii) Let $G_{-} G_{+}$be another (canonical) Wiener-Hopf factorization of $G$, obtained e.g. by solving a Riemann-Hilbert problem with coefficient $G\left(G \Phi^{+}=\Phi^{-}\right)$. Then we have

$$
G=G_{-} G_{+}=\widetilde{G}_{-} \widetilde{G}_{+} \text {. }
$$


In the above relation both factorizations have factors that with their inverses are bounded analytic in their domains of existence, $G_{-}, G_{+}$, because it is assumed to be a Wiener-Hopf factorization and $\widetilde{G}_{-}, \widetilde{G}_{+}$because the factors are assumed to satisfy equations (2.6).

From (2.9) we have

$$
\widetilde{G}_{-}^{-1} G_{-}=\widetilde{G}_{+} G_{+}^{-1}
$$

which implies that both sides equal a constant in $\lambda$, but since we have a relation (2.10) for each $t$, both sides must equal a function of $t$, independent of $\lambda$. We write

$$
\widetilde{G}_{-}^{-1} G_{-}=\widetilde{G}_{+} G_{+}^{-1}=F_{t}
$$

i.e.

$$
\widetilde{G}_{-}=G_{-} F_{t}^{-1}, \quad \widetilde{G}_{+}=F_{t} G_{+} .
$$

$F_{t}$ plays the role of a normalization factor at the point $t$ for $G_{-}, G_{+}$.

Theorem 2.7. Let $F_{t}$ satisfy the linear differential equation

$$
\frac{d F_{t}}{d t}=A_{0} F_{t} .
$$

Then the function $L_{t}$ given by

$$
L_{t}=F_{t} \hat{L}_{t} F_{t}^{-1}
$$

with $\hat{L}_{t}=G_{+} L_{0} G_{+}^{-1}$, satisfies the Lax equation (2.2). Here $G_{+}$is as in Theorem 2.6 (as are $G_{-}, G$ ).

Proof. We show that $L_{t}$ given by (2.12) with $F_{t}$ satisfying (2.11) is a solution to equation (2.2). We have

$$
\begin{array}{r}
\frac{d L_{t}}{d t}=A_{0} F_{t} \hat{L}_{t} F_{t}^{-1}+F_{t} \frac{d \hat{L}_{t}}{d t} F_{t}^{-1}-F_{t} \hat{L}_{t} F_{t}^{-1} A_{0} F_{t} F_{t}^{-1} \\
=\left[A_{0}, L_{t}\right]+F_{t} \frac{d \hat{L}_{t}}{d t} F_{t}^{-1} .
\end{array}
$$

Since $\hat{L}_{t}=G_{+} L_{0} G_{+}^{-1}$ it satisfies a standard Lax equation

$$
\frac{d \hat{L}_{t}}{d t}=\left[\hat{L}_{t}^{+}, \hat{L}_{t}\right]
$$

(see e.g. [4, proof of Theorem 2.7]). Hence, noting that $L_{t}=F_{t} \hat{L}_{t} F_{t}^{-1}$, we get

$$
\frac{d L_{t}}{d t}=\left[L_{t}^{+}+A_{0}, L_{t}\right] .
$$

which is equation (2.2). 
Proposition 2.8. Equation (2.11) is equivalent to the equation in $\Omega$

$$
\frac{d F_{t}}{d t}=F_{t} \hat{A}_{0}
$$

where $\hat{A}_{0}=P_{0} \hat{L}_{t}=P_{0} G_{+} L_{0} G_{+}^{-1}$.

Proof. Let $A_{0}=P_{0} L_{t}$ where $P_{0}$ is a linear bounded operator as assumed in the definition of the right-hand side of equation (2.2). Define

$$
\hat{A}_{0}=F_{t}^{-1} A_{0} F_{t} \text {. }
$$

Noting that $F_{t}$ is independent of $\lambda, F_{t}$ commutes with $P_{0}$ which leads to

$$
\hat{A}_{0}=P_{0} F_{t}^{-1} L_{t} F_{t}=P_{0} \hat{L}_{t}=P_{0} G_{+} L_{0} G_{+}^{-1} .
$$

Introducing in equation (2.11) the definition (2.14) of $\hat{A}_{0}$ we obtain

$$
\frac{d F_{t}}{d t}=F_{t} \hat{A}_{0} F_{t}^{-1} F_{t}=F_{t} \hat{A}_{0}
$$

as required.

Remark 2.9. Equation (2.13) is more convenient for calculating the solution of equation (2.2) since $\hat{A}_{0}$ is known explicitly whereas $A_{0}$ is not.

Proposition 2.10. $G=\exp \left(t L_{0}\right)$ has a canonical factorization at a point $t_{i} \in \Omega$ with factors differentiable w.r.t. $t$ in a neighborhood of $t_{i}$ iff equation (2.2) has a solution at the point $t_{i}$.

Proof. Sufficiency: Assume that equation (2.2) has a solution at a point $t_{i} \in \Omega$. Then by Theorem 2.3 there exist functions $\widetilde{G}_{-}, \widetilde{G}_{+}$satisfying (2.6) in a neighborhood of $t_{i}$ ( $\Omega$ is open) which give the solution to equation (2.2),

$$
L_{t}=\widetilde{G}_{+} L_{0} \widetilde{G}_{+}^{-1}=\widetilde{G}_{-}^{-1} L_{0} \widetilde{G}_{-} .
$$

From Theorem $2.6 \widetilde{G}_{+}, \widetilde{G}_{-}$are related to the factors of another canonical factorization of $G\left(G_{-}, G_{+}\right)$by the formulas

$$
G_{-}=\widetilde{G}_{-} F_{t}, \quad G_{+}=F_{t}^{-1} \widetilde{G}_{+}
$$

where $F_{t}$ satisfies the differential equation (2.11). Since $\widetilde{G}_{-}, \widetilde{G}_{+}$and $F_{t}$ are differentiable in a vicinity of $t_{i}$ it follows that the factors $G_{-}$, $G_{+}$are differentiable too.

Necessity: Assume that the factors $G_{-}, G_{+}$of a canonical factorization of $G$ are differentiable. Then

$$
\frac{d G}{d t}=L_{0} G=\frac{d G_{-}}{d t} G_{+}+G_{-} \frac{d G_{+}}{d t}
$$

and letting $\hat{L}_{t}=G_{-}^{-1} L_{0} G_{-}$we get from the above relation

$$
\hat{L}_{t}^{+}:=P^{+} \hat{L}_{t}=\frac{d G_{+}}{d t} G_{+}^{-1},
$$


and, putting $L_{t}=F_{t} \hat{L}_{t} F_{t}^{-1}$ with $F_{t}$ satisfying (2.11), we have (see the proof of Theorem 2.7)

$$
\frac{d L_{t}}{d t}=\left[L_{t}^{+}+A_{0}, L_{t}\right]
$$

which is equation (2.2).

\section{Singularities via the Riemann-Hilbert approach}

In this section we present our main result which enables us to locate the singularities of the solution to equation (2.2) without obtaining the explicit solution of the associated Riemann-Hilbert problem. Here the use of the factorization method is crucial since it allows us to translate the problem of the existence of singularities into an operator theory problem.

We recall from the Introduction the direct sum decomposition of $\left[C_{\mu}\left(S^{1}\right)\right]^{n}$

$$
\left[C_{\mu}\left(S^{1}\right)\right]^{n}=\left[C_{\mu}^{+}\right]^{n} \oplus\left[C_{\mu}^{-}\right]_{0}^{n},
$$

where $\left[C_{\mu}^{+}\right]^{n}$ denotes the subspace of $\left[C_{\mu}\left(S^{1}\right)\right]^{n}$ of functions analytic in $\mathbb{D}$ and $\left[C_{\mu}^{-}\right]_{0}^{n}$ is the subspace of analytic functions in $\mathbb{C} \backslash \overline{\mathbb{D}}$ that vanish at infinity. We let $P^{+}:\left[C_{\mu}\left(S^{1}\right)\right]^{n} \rightarrow\left[C_{\mu}^{+}\right]^{n}$ denote the projection associated to this decomposition (so that $\operatorname{ker} P^{+}=\left[C_{\mu}^{-}\right]_{0}^{n}$ ).

Given a matrix $G \in\left[C_{\mu}\left(S^{1}\right)\right]^{n \times n}$ the corresponding multiplication operator in $\left[C_{\mu}^{+}\right]^{n}$ is denoted $G I_{+}$. The composite

$$
P^{+} G I_{+}:\left[C_{\mu}^{+}\right]^{n} \rightarrow\left[C_{\mu}^{+}\right]^{n}
$$

is a Topelitz operator (with symbol $G[6$, Ch.1]) whose properties are closely related to those of its symbol $G$. We recall from [6, Ch.1] that the operator $\mathrm{P}^{+} G I_{+}$is invertible iff $G$ has a canonical Wiener-Hopf factorization

$$
G=G_{-} G_{+},
$$

with $\left(G^{ \pm}\right)^{ \pm 1} \in\left[C_{\mu}^{ \pm}\left(S^{1}\right)\right]$.

We are now ready to state the main result of this section.

Theorem 3.1. Let $T_{G}$ be the Toeplitz operator $P^{+} G I_{+}$defined above, where $G=\exp \left(t L_{0}\right)$.

Then equation (2.2) has a solution in a neighborhood of a point $t=t_{i}$ iff the operator $T_{G}$ is injective at that point, i.e., $\operatorname{ker} T_{G}$ is trivial.

Proof. Sufficiency: We begin by proving that if $\operatorname{ker} T_{G}$ is trivial $T_{G}$ is invertible. Firstly we note that $G=\exp \left(t L_{0}\right) \in C_{\mu}\left(S^{1}\right)$ (in fact $\left.G \in C^{\infty}\left(S^{1}\right)\right)$ for every $t \in \mathbb{C}$. Thus a factorization of $G$ of the general form

$$
G=G_{-} D G_{+}, \quad\left(G_{ \pm}\right)^{ \pm 1} \in\left[C_{\mu}^{ \pm}\left(S^{1}\right)\right]^{n \times n},
$$


where $D$ is a diagonal nonsingular rational matrix, exists for all $t \in \mathbb{C}$ (see e.g. [6, Ch.1]).

Since $G \in\left[C_{\mu}\left(S^{1}\right)\right]^{n \times n}, \operatorname{det} G \in C_{\mu}\left(S^{1}\right)$ and

$$
\operatorname{det} G=\exp \left(\left(\operatorname{tr} L_{0}\right) t\right) \neq 0,
$$

for all $\lambda \in S^{1}$, it follows that $T_{G}$ is Fredholm of zero index. This means that

$$
\operatorname{codimim} T_{G}=\operatorname{dim} \operatorname{ker} T_{G} .
$$

It follows that, if $\operatorname{ker} T_{G}$ is trivial at $t=t_{i}, T_{G}$ is invertible at this point. It is easy to see that this is true in a neighborhood of $t_{i}$. Hence $G$ has a canonical factorization

$$
G=\exp \left(t L_{0}\right)=G_{-} G_{+}
$$

in a neighborhood of $t_{i}$. By Proposition 2.10 this implies that equation (2.2) has a solution at this point.

Necessity: Assume that a solution to equation (2.2) exists at $t=t_{i}$. Then, by Proposition 2.10, $G$ possesses a canonical factorization at $t=t_{i}$, i.e.,

$$
G=G_{-} G_{+}, \quad\left(G_{ \pm}\right)^{ \pm 1} \in\left[C_{\mu}^{ \pm}\left(S^{1}\right)\right]^{n \times n} .
$$

This is equivalent to the invertibility of $T_{G}$ in a neighborhood of $t_{i}$ and thus $\operatorname{ker} T_{G}$ is trivial.

In the next two propositions we express the condition of Theorem 3.1 in terms of the existence of solutions to a certain Riemann-Hilbert problem, which has the advantage of being easier to analyse.

Proposition 3.2. Let $T_{G}$ be the operator defined in Theorem 3.1. Then $\operatorname{ker} T_{G}$ is nontrivial iff the Riemann-Hilbert problem

$$
G \Phi^{+}=\Phi^{-}, \quad \Phi^{ \pm} \in\left[C_{\mu}^{ \pm}\left(S^{1}\right)\right]^{n},
$$

with $\Phi^{-}(\infty)=0$, has non-trivial solutions.

Proof. $\operatorname{ker} T_{G}$ being non-trivial means that the equation

$$
P^{+} G \Phi^{+}=0, \quad \Phi^{+} \in\left[C_{\mu}^{+}\right]^{n}
$$

has non-trivial solutions. Keeping in mind the direct sum decomposition (3.1), we see that this is equivalent to saying that the RiemannHilbert problem in $\left[C_{\mu}\left(S^{1}\right)\right]^{n}$

$$
G \Phi^{+}=\Phi^{-} \quad \text { with } \quad \Phi^{-}(\infty)=0,
$$

has non-trivial solutions.

Proposition 3.3. Let $n=2$ in Proposition 3.2. Then the vector valued Riemann-Hilbert problem (on the Riemann sphere)

$$
G \Phi^{+}=\Phi^{-}, \quad \Phi^{-}(\infty)=0,
$$


given in Proposition 3.2 is equivalent to a scalar Riemann-Hilbert problem of the form

$$
g \Psi^{+}=\Psi^{-}
$$

on a compact Riemann surface $\Sigma$ defined by the equation $\operatorname{det}\left(\mu I_{2}-\right.$ $L(\lambda))=0$ with $\Psi^{-}$subject to the condition

$$
\Psi^{-}\left(\infty_{1}\right)=0, \Psi\left(\infty_{2}\right)=0
$$

where $\infty_{1}, \infty_{2}$ are the poles of the meromorphic function given by the projection

$$
\Sigma \rightarrow \mathbb{P}^{1}(\mathbb{C}),(x, w) \mapsto x
$$

(i.e., $\infty_{1}, \infty_{2}$ are the points of $\Sigma$ "at infinity").

Proof. It is proven in [5] that the Riemann-Hilbert problem (3.2) is equivalent, for $n=2$, to a scalar Riemann-Hilbert problem on $\Sigma(3.3)$. The condition (3.4) is the translation of the condition $\Phi^{-}(\infty)=0$ in (3.2) to the Riemann surface.

\section{ExAmple}

In this section we study a dynamical system for which the solution and, consequently, its singularities can be obtained by classical methods and compare the result obtained with that given by the method of Section 3 .

4.1. Dynamical system. We take the example presented in [4] which is given by the equations

$$
\frac{d L_{t}}{d t}=\left[L_{t}^{+}, L_{t}\right]
$$

where

$$
L_{t}(\lambda)=\left[\begin{array}{rr}
v(\lambda) & u(\lambda) \\
w(\lambda) & -v(\lambda)
\end{array}\right], \quad \lambda \in S^{1}
$$

with

$$
\begin{aligned}
v(\lambda) & =z \lambda^{-1} \\
u(\lambda) & =a \lambda+y \lambda^{-1}+x, \quad a \in \mathbb{C} \\
w(\lambda) & =a \lambda+y \lambda^{-1}-x
\end{aligned}
$$

and $L_{t}^{+}$being the polynomial part of $L_{t}$ (with respect to $\lambda$ ). It can easily be seen that equation (4.1) together with (4.2) and (4.3) is equivalent to the following nonlinear system of differential equations

$$
\frac{d x}{d t}=-2 a z, \quad \frac{d y}{d t}=-2 x z, \quad \frac{d z}{d t}=2 x y
$$

for the dynamical variables $x, y, z$. This system admits two integrals of the motion, namely,

$$
A=x^{2}-2 a y, \quad B=y^{2}+z^{2} .
$$


That these are invariants is easily checked by differentiating both sides of relations (4.5) and using equations (4.4).

4.2. Classical solution. To obtain an equation of the movement in the variable $x$ we begin with the first of equations (4.4)

$$
(\dot{x})^{2}=4 a^{2} z^{2}=4 a^{2}\left(B-y^{2}\right)
$$

where $\dot{x}=\frac{d x}{d t}$. Using relations (4.5) yields

$$
(\dot{x})^{2}=4 a^{2} B-\left(A-x^{2}\right)^{2}=4 a^{2} B-A^{2}+2 A x^{2}-x^{4}
$$

from which we get

$$
\dot{x}=\mathrm{i} \sqrt{p(x)}
$$

where $p(x)=x^{4}-2 A x^{2}+A^{2}-4 a^{2} B$. The above equation means that $(\dot{x}, x)$ lies in an elliptic curve, i.e., the orbits of the dynamics lie in an elliptic Riemann surface.

Before we integrate (4.6) we note that if we derive equations for the variables $y, z$ we obtain equation (4.6) after an elementary transformation on these variables as was to be expected.

Integration of (4.6) gives

$$
\int_{x_{0}}^{x} \frac{d x}{\sqrt{p(x)}}=\mathrm{i} t
$$

where $x_{0}$ is the value of $x$ at $t=0$, and the path of integration is understood to be on the Riemann surface $\Sigma$ defined by

$$
w^{2}=p(x)=\left(x^{2}-x_{1}^{2}\right)\left(x^{2}-x_{2}^{2}\right)
$$

with the zeros of $p(x), \pm x_{1}, \pm x_{2}$, given by

$$
x_{1}^{2}=A+2 a \sqrt{B}, \quad x_{2}^{2}=A-2 a \sqrt{B} .
$$

It is useful to write (4.8) in the normalized form

$$
w^{2}=x_{1}^{2} x_{2}^{2}\left(1-\widetilde{x}^{2}\right)\left(1-k^{2} \widetilde{x}^{2}\right)
$$

where $\widetilde{x}=x / x_{1}$ and $k^{2}=x_{1}^{2} / x_{2}^{2}$. From now on we take as a definition of the Riemann surface $\Sigma$ the following equation

$$
w^{2}=\left(1-x^{2}\right)\left(1-k^{2} x^{2}\right),
$$

which corresponds to making the change of variables $x \mapsto x / x_{1}, w \mapsto$ $w /\left(x_{1} x_{2}\right)$. With this notation, (4.7) takes the form

$$
\int_{\widetilde{x}_{0}}^{\widetilde{x}} \frac{d x}{w(x)}=\mathrm{i} x_{2} t \quad(\text { on } \Sigma \text { 2 })
$$


with $\widetilde{x}=x / x_{1}, \widetilde{x}_{0}=x_{0} / x_{1}$. It is convenient to write the above integral as a difference of two integrals as follows

$$
\mathrm{i} x_{2} t=\int_{0}^{\widetilde{x}} \frac{d x}{w(x)}-\int_{0}^{\widetilde{x}_{0}} \frac{d x}{w(x)} \quad(\text { on } \Sigma)
$$

We are looking for the singularities of the solution to equation (4.6) so we let $x \rightarrow \infty$ which leads to

$$
\int_{0}^{\widetilde{x}} \frac{d x}{w(x)} \rightarrow \mathrm{i} \mathbf{K}^{\prime} \quad \text { or } \quad \int_{0}^{\widetilde{x}} \frac{d x}{w(x)} \rightarrow \mathrm{i} \mathbf{K}^{\prime}+2 \mathbf{K},
$$

where $\mathbf{K}, \mathbf{K}^{\prime}$ are, respectively, the complete elliptic integral and the complementary complete elliptic integral of the first kind (see e.g. [2]). Hence from (4.11), keeping in mind that (4.11) is an equation on $\Sigma$, we obtain

$$
\mathrm{i} x_{2} t=\mathrm{i} \mathbf{K}^{\prime}-u_{0}+4 m \mathbf{K}+2 \mathrm{i} \mathbf{K}^{\prime}
$$

or

$$
\mathrm{i} x_{2} t=\mathrm{i} \mathbf{K}^{\prime}+2 \mathbf{K}-u_{0}+4 m \mathbf{K}+2 \mathrm{i} n \mathbf{K}^{\prime}, \quad n, m \in \mathbb{Z},
$$

where

$$
u_{0}=\int_{0}^{\widetilde{x}_{0}} \frac{d x}{w(x)} .
$$

The above formulas for $t$ are equivalent to the single formula

$$
\mathrm{i} x_{2} t=-u_{0}+\mathrm{i} \mathbf{K}^{\prime}+2 m \mathbf{K}+2 \mathrm{i} n \mathbf{K}^{\prime}, \quad n, m \in \mathbb{Z} .
$$

This relation gives us the values of $t$ at which singularities occur, i.e., where the solution blows up.

4.3. Riemann-Hilbert solution. Next we derive a formula for the singularities of the solution to system (4.4) using the method of Propositions 3.2 and 3.3 . To this end we need to formulate the RiemannHilbert problem (3.2) for the function $G=\exp \left(t L_{0}\right)$ in an associated Riemann surface. Recalling (3.2) we have

$$
G \Phi^{+}=\Phi^{-} \quad \text { with } \quad G=\exp \left(t L_{0}\right),
$$

and $\Phi^{ \pm} \in\left[C_{\mu}^{ \pm}\right]^{2}$ with the condition $\Phi^{-}(\infty)=0$.

Taking into account that $L_{0}$ can be diagonalized as

$$
L_{0}=S D_{0} S^{-1}
$$

with

$$
S=\left[\begin{array}{cc}
1 & -1 \\
\frac{\mu-z_{0}}{q_{1}(\lambda)} & \frac{\mu+z_{0}}{q_{1}(\lambda)}
\end{array}\right] \quad\left(q_{1}(\lambda):=a \lambda^{2}+x_{0} \lambda+y_{0}\right)
$$

\footnotetext{
${ }^{2}$ We identity $\Sigma$ with the quotient of $\mathbb{C}$ by the lattice of periods of $d x / \sqrt{p(x)}$.
} 
and $D_{0}=\operatorname{diag}\left(\lambda^{-1} \mu,-\lambda^{-1} \mu\right)$ where $\mu=\lambda \nu$ with $\nu$ given by the characteristic equation of $L_{0}$,

$$
\operatorname{det}\left(\nu I_{2}-L_{0}(\lambda)\right)=0 .
$$

From this equation we obtain

$$
\mu^{2}=p_{1}(\lambda):=a^{2} \lambda^{4}-\left(x_{0}^{2}-2 a y_{0}\right) \lambda^{2}+z_{0}^{2}+y_{0}^{2}
$$

or, introducing the invariants $A$ and $B$,

$$
p_{1}(\lambda)=a^{2} \lambda^{4}-A \lambda^{2}+B .
$$

The explicit formulas for the zeros of $p_{1}(\lambda), \pm \lambda_{1}, \pm \lambda_{2}$, are given in (4.33) below. Relation (4.18) defines an elliptic Riemann surface, which is associated with $L_{0}$ (or $L_{t}$ as it is independent of the dynamics). We denote by $\Sigma_{1}$ the compact Riemann surface obtained by adding two points at infinity $\infty_{1}, \infty_{2}$.

Going back to (4.15) it follows from it that

$$
G=\exp \left(t L_{0}\right)=S D S^{-1}
$$

where $D=\operatorname{diag}\left(\exp \left(t \lambda^{-1} \mu\right), \exp \left(-t \lambda^{-1} \mu\right)\right)$.

Hence (4.14) may be written as

$$
D S^{-1} \Phi^{+}=S^{-1} \Phi^{-}
$$

which, in terms of the components of $\Phi^{ \pm}$, denoted $\left(\phi_{1}^{ \pm}, \phi_{2}^{ \pm}\right)$, is written as

$$
\begin{cases}d_{1}\left(z_{0} \phi_{1}^{+}+q_{1} \phi_{2}^{+}+\mu \phi_{1}^{+}\right) & =z_{0} \phi_{1}^{-}+q_{1} \phi_{2}^{-}+\mu \phi_{1}^{-} \\ d_{2}\left(z_{0} \phi_{1}^{+}+q_{1} \phi_{2}^{+}-\mu \phi_{1}^{+}\right) & =z_{0} \phi_{1}^{-}+q_{1} \phi_{2}^{-}-\mu \phi_{1}^{-}\end{cases}
$$

where $d_{1}=\exp \left(t \lambda^{-1} \mu\right), d_{2}=\exp \left(-t \lambda^{-1} \mu\right)$.

The above system is equivalent to the following single scalar equation (for more details see [4] or [5]) on a contour $\Gamma$ that is the preimage of $S^{1}$ under the projection $\varrho:(\lambda, \mu) \mapsto \lambda$,

$$
d\left(\phi_{2}^{+}+\frac{z_{0}+\mu}{q_{1}} \phi_{1}^{+}\right)=\phi_{2}^{-}+\frac{z_{0}+\mu}{q_{1}} \phi_{1}^{-} .
$$

Note that $\Gamma$ has two connected components; we put $d=d_{1}$ on one of these components and $d=d_{2}$ on the other. In view of the expressions for $d_{1}$ and $d_{2}$ we have

$$
d=\exp \left(\frac{\mu}{\lambda} t\right), \quad(\lambda, \mu) \in \Gamma .
$$

Concerning equation (4.21), it is also useful to note that setting $q_{2}(\lambda)=a \lambda_{2}-x_{0} \lambda+y_{0}$, we have

$$
\mu^{2}-z_{0}^{2}=q_{1}(\lambda) q_{2}(\lambda)
$$

It follows that, as a meromorphic function on $\Sigma_{1}, q_{1}(\lambda)$ has four zeros, two of which are zeros of $\mu+z_{0}$ and the other two are zeros of $\mu-z_{0}$. 
To solve (4.21) we note that $d$ can be factorized on the Riemann surface as

$$
d=d_{-} r d_{+},
$$

where $\left(d^{+}\right)^{ \pm 1} \in C_{\mu}(\Gamma)$ extends holomorphically to the preimage $\Omega^{+}$ of $\mathbb{D}$ under the projection $\varrho$ and, similarly, $\left(d^{-}\right)^{ \pm 1} \in C_{\mu}(\Gamma)$ admits a holomorphic extension to the preimage $\Omega^{-}$of $\mathbb{P}(\mathbb{C}) \backslash \overline{\mathbb{D}}$. Finally, $r$ is a rational function on $\Sigma_{1}$. See [4] or [5] for more details.

Note that all three factors in the above factorization depend on $t$. Introducing this factorization in (4.21), we get

$$
r d_{+}\left(\phi_{2}^{+}+\frac{z_{0}+\mu}{q_{1}} \phi_{1}^{+}\right)=d_{-}^{-1}\left(\phi_{2}^{-}+\frac{z_{0}+\mu}{q_{1}} \phi_{1}^{-}\right)=R,
$$

where $R$ is a rational function on $\Sigma_{1}$.

For the computations that follow it is convenient to rewrite the Riemann surface $\Sigma_{1}$ using the normalized equation

$$
\mu^{2}=\left(1-\lambda^{2}\right)\left(1-k_{1}^{2} \lambda^{2}\right)
$$

where $k_{1}=\lambda_{1} / \lambda_{2}$ and $\pm \lambda_{1}, \pm \lambda_{2}$ are the roots of $p_{1}(\lambda)(c f$. (4.18) $)$ given in (4.33) below. This corresponds to making a change of variables $\lambda \mapsto \lambda / \lambda_{1}, \mu \mapsto \mu /\left(a \lambda_{1} \lambda_{2}\right)$.

Also, from now on we identify $\Sigma_{1}$ with its Jacobian, using the Abel map

$$
(\lambda, \mu) \mapsto u=\int_{0}^{(\lambda, \mu)} \frac{d \lambda}{\mu},
$$

i.e., we consider all equations relating points of $\Sigma_{1}$ as written on the quotient of $\mathbb{C}$ by the lattice of periods of the holomorphic form $d \lambda / \mu$.

Hence, keeping in mind that $\Sigma_{1}$ is an elliptic Riemann surface $\left(p_{1}\right.$ is a fourth degree polynomial), $R$ can be expressed in elliptic theta functions. To this end we recall that we are solving (4.24) with the conditions $\phi_{i}^{-}\left(\infty_{j}\right)=0$, for $i, j=1,2$, where $\infty_{1}, \infty_{2}$ are the two points at infinity 3 in $\Sigma_{1}$, which correspond to $\infty$ under the projection $\varrho:(\lambda, \mu) \mapsto \lambda$. Denoting by $\psi^{+}, \psi^{-}$the expression within parentheses in both sides of (4.24), these conditions correspond to

$$
\psi^{-}\left(\infty_{1}\right)=0, \quad \psi^{-}\left(\infty_{2}\right)=0 .
$$

Before introducing these conditions we note that, using the Jacobi theta function $\vartheta_{1}$ that satisfies $\vartheta_{1}(0)=0, R$ has the expression

$$
R(u)=\gamma \frac{\vartheta_{1}\left(u-v_{0}\right) \vartheta_{1}\left(u-v_{1}\right) \vartheta_{1}\left(u-v_{2}\right)}{\vartheta_{1}\left(u-u_{0}\right) \vartheta_{1}\left(u-u_{1}\right) \vartheta_{1}\left(u-u_{2}\right)}
$$

where $\gamma \in \mathbb{C}$ and the zeros and poles of $R$ are determined by the following conditions:

\footnotetext{
${ }^{3}$ We choose $\infty_{1}$ such that $\mu \sim k_{1} \lambda^{2}$ near $\infty_{1}$.
} 
(i) $R$ has a pole at the point $u_{0}$ corresponding to the pole of $r$ in $\Omega^{+}$ (see [4, Appendix B]);

(ii) $R$ has a zero at the point $v_{0}$ corresponding to the zero of $r$ in $\Omega^{+}$ (see [4, Appendix B]);

(iii) $R$ has two poles $u_{1}, u_{2}$ at the zeros of $q_{1}$ that do not coincide with the zeros of $a \lambda_{1} \lambda_{2} \mu+z_{0}$ (which is $\mu+z_{0}$ in (4.24) written in the normalized coordinates of (4.25) );

(iv) $R$ has two zeros at points $v_{1}, v_{2}$ imposed by condition (4.27), i.e.,

$$
v_{1}=\infty_{1}, \quad v_{2}=\infty_{2} .
$$

(v) The zeros and poles of $R$ must satisfy Abel's condition:

$$
v_{0}-u_{0}=u_{1}+u_{2}-\infty_{1}-\infty_{2} \quad\left(\bmod 2 \mathrm{i} \mathbf{K}_{1}^{\prime}+4 \mathbf{K}_{1}\right),
$$

where $\mathbf{K}_{1}$ and $\mathbf{K}_{1}^{\prime}$ are the complete elliptic and complementary elliptic integrals of the first kind of $\Sigma_{1}$.

From the analysis of the factorization of the function $d$ given in (4.22) (see [4, Definition B.7 and Proposition B.9]) we obtain

$$
v_{0}-u_{0}=2 a t \lambda_{2}
$$

where $\lambda_{2}$ is as in the expression for $p_{1}(\lambda)$ (see text following (4.19)). Substitution of (4.30) in (4.29) gives us the expression for the values of $t$ for which singularities occur. Taking into account that $\infty_{1}+\infty_{2}=$ $2 \mathbf{K}_{1}+2 \mathrm{i} \mathbf{K}_{1}^{\prime}\left(\bmod 4 m \mathbf{K}_{1}+2 \mathrm{i} n \mathbf{K}_{1}^{\prime}\right)$ we have

$$
2 a t \lambda_{2}=u_{1}+u_{2}+2 \mathbf{K}_{1}+4 m \mathbf{K}_{1}+2 \mathrm{i} n \mathbf{K}_{1}^{\prime}
$$

where $u_{1}, u_{2}$ are the images under Abel's map of the zeros of $q_{1}$ that do not coincide with zeros of $a \lambda_{1} \lambda_{2} \mu+z_{0}$, i.e.,

$$
u_{1}=\int_{0}^{\hat{\lambda}_{1} / \lambda_{1}} \frac{d \lambda}{\mu}, \quad u_{2}=\int_{0}^{\hat{\lambda}_{2} / \lambda_{1}} \frac{d \lambda}{\mu}
$$

where $\hat{\lambda}_{1}, \hat{\lambda}_{2}$ are de zeros of $q_{1}(\lambda)$ and $\lambda_{1}$ is a zero of $p_{1}(\lambda)$ given in (4.33) below.

Thus (4.31) gives us the values of $t$ leading to singularities of the solution of Lax equation (2.2) as derived from the theory of Section 3 .

Remark 4.1. (i) Formula (4.31) was obtained without requiring an explicit formula for $\phi_{1}^{ \pm}, \phi_{2}^{ \pm}$corresponding to the factors of the canonical factorization of $G, G=G_{-} G_{+}$, although these functions can easily be obtained from (4.24), replacing condition (iv) by the imposition of a zero at a chosen point $v_{1}$. Then Abel's condition $(v)$ gives the zero $v_{2}$ (see [4] for the details). The solution thus obtained gives the factors $G_{-}, G_{+}$of $G$ providing $t$ does not satisfy (4.31), a result that could not be obtained in [4].

(ii) Formulas (4.31) and (4.13) are not easily compared since they involve different Riemann surfaces. The appearance of distinct 
surfaces when using different methods to study integrable systems is an intriguing phenomenon that occurs in other examples [3].

We show next that the two Riemann surfaces are closely related and that the two expressions for the singularities coincide.

4.4. Comparison of solutions. We start by showing that the two Riemann surfaces $\Sigma$ and $\Sigma_{1}$ are related and this will enable us to express both formulas (4.13) and (4.31) on the same Riemann surface thus allowing for a comparison of the two results.

The Riemann surface $\Sigma$ is defined by the equation

$$
w^{2}=\left(1-x^{2}\right)\left(1-k^{2} x^{2}\right)=\frac{p\left(x_{1} x\right)}{\left(x_{1} x_{2}\right)^{2}}
$$

with $p(x)=x^{4}-2 A x^{2}+A^{2}-4 a^{2} B=\left(x^{2}-x_{1}^{2}\right)\left(x^{2}-x_{2}^{2}\right)$ and $k=x_{1} / x_{2}$, where

$$
x_{1}^{2}=A+2 a \sqrt{B}, \quad x_{2}^{2}=A-2 a \sqrt{B} .
$$

The Riemann surface $\Sigma_{1}$ is defined by the equation

$$
\mu^{2}=\left(1-\lambda^{2}\right)\left(1-k_{1}^{2} \lambda^{2}\right)=\frac{p_{1}\left(\lambda_{1} \lambda\right)}{\left(a \lambda_{1} \lambda_{2}\right)^{2}}
$$

with $p_{1}(\lambda)=a^{2} \lambda^{4}-A \lambda^{2}+B=a^{2}\left(\lambda^{2}-\lambda_{1}^{2}\right)\left(\lambda^{2}-\lambda_{2}^{2}\right)$, where

$$
\lambda_{1}^{2}=\frac{A+\sqrt{A^{2}-4 a^{2} B}}{2 a^{2}}, \quad \lambda_{2}^{2}=\frac{A-\sqrt{A^{2}-4 a^{2} B}}{2 a^{2}} .
$$

From the expression for $p(x)$ and (4.32) we have

$$
\begin{aligned}
A^{2}-4 a^{2} B & =x_{1}^{2} x_{2}^{2} \\
2 A & =x_{1}^{2}+x_{2}^{2} .
\end{aligned}
$$

Introducing these relations in (4.33) gives

$$
\lambda_{1}^{2}=\left(\frac{x_{2}-x_{1}}{2 a}\right)^{2}, \quad \lambda_{2}^{2}=\left(\frac{x_{1}+x_{2}}{2 a}\right)^{2}
$$

which leads to

$$
\lambda_{1}=\frac{x_{2}-x_{1}}{2 a}, \quad \lambda_{2}=\frac{x_{1}+x_{2}}{2 a}
$$

where the sign in the square root is determined by a direct check on (4.33). From (4.35) we now get the relation between the moduli of the two surfaces

$$
k_{1}=\frac{\lambda_{1}}{\lambda_{2}}=\frac{x_{2}-x_{1}}{x_{2}+x_{1}}=\frac{1-k}{1+k}
$$

where $k$ and $k_{1}$ denote the elliptic moduli of $\Sigma$ and $\Sigma_{1}$, respectively (see [2]). This shows that the surfaces are closely related as claimed at the end of Section 4.3 .

Having obtained equality (4.36) we are now in a position to state the following proposition relating $\Sigma$ and $\Sigma_{1}$. 
Proposition 4.2. The following statements express the relation between the Riemann surfaces $\Sigma$ and $\Sigma_{1}$ :

(i) For the elliptic moduli of $\Sigma$ and $\Sigma_{1}$, respectively $k, k_{1}$, we have

$$
k_{1}=\frac{1-k}{1+k}
$$

(ii) There is a holomorphic map $\varphi: \Sigma \rightarrow \Sigma_{1}$ given by

$$
\Sigma \ni(x, w) \mapsto(\lambda, \mu):=\left(\mathrm{i}(1+k) \frac{x}{w}, \frac{k^{2} x^{4}-1}{w^{2}}\right) \in \Sigma_{1} .
$$

(iii) Under the map $\varphi$ of (ii) the points at infinity of $\Sigma$ are mapped to $\mathbf{o}_{1}:=(0,1)$ and $(0, \pm 1),( \pm 1,0),( \pm 1 / k, 0)$ are mapped to $\mathbf{o}_{2}:=$ $(0,-1), \infty_{1}$ and $\infty_{2}$, respectively.

(iv) The relation between the holomorphic forms of both surfaces is expressed by

$$
\varphi^{*}\left(\frac{d \lambda}{\mu}\right)=-\mathrm{i}(1+k) \frac{d x}{w} .
$$

Proof. (i) was proven in (4.36).

The formula in (ii) is obtained by composing the two Gauss transformations corresponding in terms of elliptic moduli to $k \mapsto k_{1}^{\prime}$ and $k^{\prime} \mapsto k_{1}$ (see [2, §39]). That it defines a map $\Sigma \rightarrow \Sigma_{1}$ can be directly checked by a substitution of (4.36) in $\mu^{2}=\left(1-\lambda^{2}\right)\left(1-k_{1}^{2} \lambda^{2}\right)$. We note that this map is not injective; in fact it is 2 to 1 . (iii) is easily obtained by direct substitution in formula (ii).

The expression (iv) follows directly from (ii) by differentiation.

Before we attempt to formulate expression (4.13) in $\Sigma_{1}$ we are going to write $u_{1}+u_{2}$ of (4.31) as a single integral as in (4.13) in order to make it possible to compare the two results. From (4.31)

$$
u_{1}+u_{2}=\int_{0}^{\hat{\lambda}_{1} / \lambda_{1}} \frac{d \lambda}{\mu}+\int_{0}^{\hat{\lambda}_{2} / \lambda_{1}} \frac{d \lambda}{\mu}
$$

which we seek to write in the form

$$
u_{1}+u_{2}=\int_{0}^{\xi_{0}} \frac{d \lambda}{\mu}
$$

To obtain $\xi_{0}$ we make use of the formula for the sum of arguments of the elliptic function sn (see [2]),

$$
\operatorname{sn}\left(u_{1}+u_{2}\right)=\frac{\operatorname{sn} u_{1} \operatorname{cn} u_{2} \operatorname{dn} u_{2}+\operatorname{sn} u_{2} \operatorname{cn} u_{1} \operatorname{dn} u_{1}}{1-k_{1}^{2} \operatorname{sn}^{2} u_{1} \operatorname{sn}^{2} u_{2}} .
$$


We have:

$$
\begin{aligned}
\operatorname{sn} u_{1} & =\frac{\hat{\lambda}_{1}}{\lambda_{1}}, \quad \operatorname{sn} u_{2}=\frac{\hat{\lambda}_{2}}{\lambda_{1}}, \\
\operatorname{cn} u_{2} \operatorname{dn} u_{2} & =\left[1-\left(\frac{\hat{\lambda}_{2}}{\lambda_{1}}\right)^{2}\right]^{1 / 2} \cdot\left[1-k_{1}^{2}\left(\frac{\hat{\lambda}_{2}}{\lambda_{1}}\right)^{2}\right]^{1 / 2}=\mu\left(\frac{\hat{\lambda}_{2}}{\lambda_{1}}\right), \\
\operatorname{cn} u_{1} \operatorname{dn} u_{1} & =\mu\left(\frac{\hat{\lambda}_{1}}{\lambda_{1}}\right) .
\end{aligned}
$$

Since $q_{1}\left(\lambda_{1} \lambda\right) q_{2}\left(\lambda_{1} \lambda\right)=\left(a \lambda_{1} \lambda_{2} \mu\right)^{2}-z_{0}^{2}($ see (4.23) $)$ and $\hat{\lambda}_{1}, \hat{\lambda}_{2}$ are the zeros of $q_{1}$ that are not zeros of $a \lambda_{1} \lambda_{2}+z_{0}$, we have

$$
\mu\left(\frac{\hat{\lambda}_{1}}{\lambda_{1}}\right)=\mu\left(\frac{\hat{\lambda}_{2}}{\lambda_{1}}\right)=\frac{z_{0}}{a \lambda_{1} \lambda_{2}}
$$

where the factor $1 /\left(a \lambda_{1} \lambda_{2}\right)$ comes from the normalization of $\mu$.

Using the above results in (4.38), taking into account, (4.37) gives

$$
\xi_{0}=\frac{1}{\lambda_{1}} \frac{\hat{\lambda}_{1}+\hat{\lambda}_{2}}{1-k_{1}^{2} \hat{\lambda}_{1}^{2} \hat{\lambda}_{2}^{2} / \lambda_{1}^{4}} \frac{z_{0}}{a \lambda_{1} \lambda_{2}}
$$

The denominator of the above formula can be simplified as follows

$$
1-k_{1}^{2} \frac{\hat{\lambda}_{1}^{2} \hat{\lambda}_{2}^{2}}{\lambda_{1}^{4}}=1-\frac{\hat{\lambda}_{1}^{2} \hat{\lambda}_{2}^{2}}{\lambda_{1}^{2} \lambda_{2}^{2}}
$$

since $k_{1}^{2}=\lambda_{1}^{2} / \lambda_{2}^{2}$. Using (4.39) we obtain

$$
1-\frac{\hat{\lambda}_{1}^{2} \hat{\lambda}_{2}^{2}}{\lambda_{1}^{2} \lambda_{2}^{2}}=1-\frac{y_{0}^{2}}{a^{2} \lambda_{1}^{2} \lambda_{2}^{2}}=\frac{z_{0}^{2}}{B}
$$

as $\hat{\lambda}_{1}^{2} \hat{\lambda}_{2}^{2}=y_{0}^{2} / a^{2}, \lambda_{1}^{2} \lambda_{2}^{2}=B / a^{2}$ and $B=y_{0}^{2}+z_{0}^{2}$.

Finally,

$$
\xi_{0}=\frac{x_{0}}{\lambda_{1} a} \frac{z_{0}}{a \lambda_{1} \lambda_{2}} \frac{B}{z_{0}^{2}}=\frac{x_{0}}{z_{0}} \lambda_{2}
$$

where we have used the result $\hat{\lambda}_{1}+\hat{\lambda}_{2}=x_{0} / a$.

We shall now transform the terms on the right-hand side of (4.13) into the surface $\Sigma_{1}$. We first take the expression for $u_{0}$ given in (4.12)

$$
u_{0}=\int_{0}^{x_{0} / x_{1}} \frac{d x}{w(x)}=\frac{\mathrm{i}}{1+k} \int_{0}^{\lambda_{0}} \frac{d \lambda}{\mu(\lambda)}
$$

where

$$
\lambda_{0}=\mathrm{i}(1+k) \frac{x_{0} / x_{1}}{w\left(x_{0} / x_{1}\right)}
$$


For the sake of simplicity in the calculations instead of trying to transform $\lambda_{0}$ into $\xi_{0}$ we prefer to take $\xi_{0}$ in (4.41) and transform it as follows:

$$
\begin{aligned}
\xi_{0} & =\frac{x_{0}}{z_{0}} \lambda_{2}=\frac{x_{0}}{w\left(x_{0} / x_{1}\right)} \frac{2 \mathrm{i} a}{x_{1} x_{2}} \lambda_{2} \\
& =\frac{x_{0}}{w\left(x_{0} / x_{1}\right)} \frac{2 \mathrm{i} a}{x_{1} x_{2}} \frac{x_{1}+x_{2}}{2 a} \\
& =\mathrm{i} \frac{x_{0}}{x_{1}} \frac{1}{w\left(x_{0} / x_{1}\right)}(1+k)=\lambda_{0} .
\end{aligned}
$$

where we have used formula (4.45) and the relation $w\left(x_{0} / x_{1}\right)=2 \mathrm{i} a z_{0} /\left(x_{1} x_{2}\right)$, which is a consequence of (4.4) and (4.6) for $t=0$. (4.35). To end the calculation for the comparison of formulas (4.13) and (4.31) we need to derive relations between the complete elliptic integrals on $\Sigma$ and $\Sigma_{1}$. Using (ii) and (iv) of Proposition 4.2 we have

$$
\begin{aligned}
\mathbf{K} & =\int_{0}^{1} \frac{d x}{w(x)}=\frac{1}{\mathrm{i}(1+k)} \int_{0}^{\infty} \frac{d \lambda}{\mu(\lambda)}=\frac{\mathbf{K}_{1}^{\prime}}{\mathrm{i}(1+k)} \\
\mathbf{K}^{\prime} & =\int_{0}^{\infty} \frac{d x}{w(x)}=\frac{1}{\mathrm{i}(1+k)} \int_{\mathbf{o}_{2}}^{\mathbf{o}_{1}} \frac{d \lambda}{\mu(\lambda)}=\frac{2 \mathbf{K}_{1}}{\mathrm{i}(1+k)} .
\end{aligned}
$$

Substitution of (4.44), (4.45) and (4.46) in (4.13) now gives

$$
-(1+k) x_{2} t=-i(1+k) u_{0}+2 \mathbf{K}_{1}+4 n \mathbf{K}_{1}+2 \mathrm{i} m K_{1}^{\prime} .
$$

Using (4.35) in (4.31) and the equalities $k=x_{1} / x_{2}, \lambda_{2}=\left(x_{1}+x_{2}\right) / 2 a$, we see that formulas (4.47) and (4.31) coincide.

\section{REFERENCES}

[1] Mark Adler, Pierre van Moerbeke, and Pol Vanhaecke, Algebraic integrability, Painlevé geometry and Lie algebras, Ergebnisse der Mathematik und ihrer Grenzgebiete. 3. Folge. A Series of Modern Surveys in Mathematics [Results in Mathematics and Related Areas. 3rd Series. A Series of Modern Surveys in Mathematics], vol. 47, Springer-Verlag, Berlin, 2004.

[2] N. I. Akhiezer, Elements of the theory of elliptic functions, Translations of Mathematical Monographs, vol. 79, American Mathematical Society, Providence, RI, 1990, Translated from the second Russian edition by H. H. McFaden.

[3] Michèle Audin, Spinning tops, Cambridge Studies in Advanced Mathematics, vol. 51, Cambridge University Press, Cambridge, 1996, A course on integrable systems.

[4] C. Câmara, A.F dos Santos, and P.F. dos Santos, Lax equations, factorization and Riemann-Hilbert problems, Portugaliae Mathematica 64 (2006), 509-533.

[5] M. C. Câmara, A. F. dos Santos, and Pedro F. dos Santos, Matrix RiemannHilbert problems and factorization on Riemann surfaces, J. Funct. Anal. 255 (2008), no. 1, 228-254.

[6] Kevin F. Clancey and Israel Gohberg, Factorization of matrix functions and singular integral operators, Operator Theory: Advances and Applications, vol. 3, Birkhäuser Verlag, Basel, 1981. 
[7] Martin A. Guest, Harmonic maps, loop groups, and integrable systems, London Mathematical Society Student Texts, vol. 38, Cambridge University Press, Cambridge, 1997.

[8] Andrew Pressley and Graeme Segal, Loop groups, Oxford Mathematical Monographs, The Clarendon Press Oxford University Press, New York, 1986, Oxford Science Publications.

[9] A.G. Reyman and A. Semenov-Tian-Shansky, Group-theoretic methods in the theory of finite-dimensional integrable systems, Dynamical Systems (S. Novikov V. Arnold, ed.), Encyclopedia of Mathematical Sciences, vol. VII, Springer, 1994.

[10] M. A. Semenov-Tian-Shansky, Integrable systems and factorization problems, Factorization and integrable systems (Faro, 2000), Oper. Theory Adv. Appl., vol. 141, Birkhäuser, Basel, 2003, pp. 155-218.

[11] M. A. Semenov-Tyan-Shanskiı̆, What a classical $r$-matrix is, Functional Analysis and Its Applications 17 (1983), no. 4, 259-272, Translated from Akademiya Nauk SSSR Funktsional i Prilozhen.

Departamento de Matemática, Instituto Superior Técnico, PortuGAL

E-mail address: afsantos@math.ist.utl.pt

Departamento de Matemática, Instituto Superior Técnico, PortuGAL

E-mail address: pedro.f.santos@math.ist.utl.pt 